\title{
SOME PROPERTIES OF FUNCTIONS WHICH UNIFORMIZE A CLASS OF SIMPLY CONNECTED RIEMANN SURFACES ${ }^{1}$
}

\author{
H. B. CURTIS, JR.
}

The following discussion defines a class of open simply connected Riemann surfaces, determines the type of a surface from this class, and obtains the forms and some properties of the uniformizing function and its derivative. Also, a proof is given to show that every mapping function of the form obtained defines a surface of this class.

Definition of the class of surfaces. Let $\left\{a_{n}\right\}_{n=1}^{\infty}$ and $\left\{b_{2 n-1}\right\}_{n=1}^{\infty}$ be two sequences such that for $n>0, a_{n}>0$ and $a_{2 n-1}<b_{2 n-1}$. For each sheet a copy of the $w$-sphere, let a surface $F$ of this class consist of sheets $S_{1}, S_{2}, \cdots, S_{n}, \cdots$ over the $w$-sphere where

(a) $S_{1}$ is slit along the real axis from $a_{1}$ to $b_{1}$; for $n \geqq 1, S_{2 n}$ is slit along the real axis from $-a_{2 n}$ to $-\infty$ and from $a_{2 n-1}$ to $b_{2 n-1}$; for $n \geqq 1, S_{2 n+1}$ is slit along the real axis from $-a_{2 n}$ to $-\infty$ and from $a_{2 n+1}$ to $b_{2 n+1}$.

(b) $S_{n}$ is joined to $S_{n+1}$ by connecting the slits which have one endpoint at $(-1)^{n+1} a_{n}$ to form first order branch points at the endpoints of the slits.

The uniformizing function. $F$ is simply connected and open. Hence by the General Uniformization Theorem there exists a unique function $z=\phi(w)$ which maps $F 1-1$ and conformally onto $\{|z|<R \leqq \infty\}$ where for $w=f(z)=\phi^{-1}(z), f(0)=0 \in S_{1}$ and $f^{\prime}(0)=1$.

$\alpha_{i}$ and $-\beta_{2 i-1}$ will denote the zeros of $f^{\prime}(z)$ corresponding to branch points over $(-1)^{i+1} a_{i}$ and $b_{2 i-1}$, respectively. $f\left(\gamma_{1}\right)=\infty \in S_{1}$, and $f\left(\gamma_{2 i}\right)=\infty$, a first order branch point over $\infty$ in the sheets $S_{2 i}$ and $S_{2 i+1} . f\left(\delta_{i}\right)=0 \in S_{i}, i=1,2, \cdots$.

By methods similar to those given in [4] it can be shown that $f(z)$ is real for $z$ real, and for $k \geqq 1,-\beta_{2 k+1}<-\gamma_{2 k}<-\beta_{1}<-\gamma_{1}<0<\alpha_{1}$ $<\delta_{2 k}<\alpha_{2 k}$. The image of $S_{1}$ is a simply connected region which contains the origin, is bounded by a simple closed curve $C_{1}$, and is symmetric about the real axis. For $k \geqq 2$, the image of $S_{k}$ is an annular region which is symmetric about the real axis and is bounded by two simple, nonintersecting closed curves $C_{k-1}$ and $C_{k}$. For $k$ odd, $C_{k}$ intersects the real axis at $-\beta_{k}$ and $\alpha_{k}$. For $k$ even, $C_{k}$ intersects the real axis at $-\gamma_{k}$ and $\alpha_{k}$.

Received by the editors November 3, 1958.

1 This is a condensation of a portion of the author's doctoral dissertation written at The Rice Institute under the direction of Professor G. R. MacLane. 
Lemma 1. If $F_{n}$ is the surface formed by the first $2 n+2$ sheets of $F$ with the slit from $-a_{2 n+1}$ to $-\infty$ deleted, then there exists a rational function which maps the z-plane onto $F_{n}$.

Proof. $F_{n}$ is a simply connected closed surface with branch points over $a_{i}$ and $b_{i}, i=1,2, \cdots, 2 n+1$, and with $n$ branch points over $\infty$. $F_{n}$ has $2 n+2$ points over the origin and two points over $\infty$ which are not branch points. Then $F_{n}$ is the Riemann surface of the inverse of a unique rational function $w=R_{n}(z)$ such that $R_{n}(0)=0 \in S_{1}$, $R_{n}^{\prime}(0)=1$, and $R_{n}(\infty)=\infty \in S_{2 n+2}$. $-\gamma_{1, n}$ and $\infty$ will denote the values of $z$ where $R_{n}(z)$ has its simple poles and $-\gamma_{2, n},-\gamma_{4, n}, \cdots$, $-\gamma_{2 n, n}$ will be the values of $z$ for double poles. If $0, \delta_{2, n}, \cdots, \delta_{2 n+2, n}$ are the zeros of $R_{n}(z)$ while $\alpha_{i, n}$ and $-\beta_{i, n}, i=1,2, \cdots, 2 n+1$, are the zeros of $R_{n}^{\prime}(z)$, then

$$
R_{n}(z)=\left[z /\left(1+z / \gamma_{1, n}\right)\right] \prod_{k=1}^{n}\left[\left(\delta_{2 k, n}^{*}\right)\left(\delta_{2 k+1, n}^{*}\right) /\left(\gamma_{2 k, n}^{*}\right)^{2}\right]\left(1-z / \delta_{2 n+2, n}\right),
$$

and

$$
R_{n}^{\prime}(z)=\left[1 /\left(1+z / \gamma_{1, n}\right)^{2}\right]\left[\prod_{k=1}^{2 n+1}\left(\alpha_{k, n}^{*}\right) \prod_{k=0}^{n}\left(\beta^{*}{ }_{2 k+1, n}\right) / \prod_{k=1}^{n}\left(\gamma_{2 k, n}^{*}\right)^{3}\right],
$$

where $\delta_{2 k, n}^{*}=1-z / \delta_{2 k, n}, \delta_{2 k+1, n}^{*}=1-z / \delta_{2 k+1, n}, \gamma_{2 k, n}^{*}=1-z / \gamma_{2 k, n}, \alpha_{k, n}^{*}=1$ $-z / \alpha_{k, n}$, and $\beta_{2 k+1, n}^{*}=1-z / \beta_{2 k+1, n}$.

Lemma 2. For $k=1,2, \cdots, 2 n+1, \alpha_{k, n}>k a_{1}$.

Lemma 3. $F$ is parabolic.

Lemma 4. As $n \rightarrow \infty, R_{n}(z) \rightarrow f(z)$ uniformly on any compact subset of the plane.

Lemma 5. For all $k \geqq 1, \alpha_{k, n} \rightarrow \alpha_{k}, \beta_{2 k-1, n} \rightarrow \beta_{2 k-1}, \gamma_{2 k, n} \rightarrow \gamma_{2 k}$, and $\delta_{k, n} \rightarrow \delta_{k}$, as $n \rightarrow \infty$.

Proof. These lemmas are proved in essentially the same way as similar results are obtained in [4].

Lemma 6. $\lim \sup _{j \rightarrow \infty} \sum_{k=1}^{j} 1 / d_{k, n}<\infty$ and $\sum_{k=2}^{\infty} 1 / d_{k}<\infty$ for the following cases: $d_{k, n}=\alpha_{k, n}$ with $j=2 n+1 ; d_{k, n}=\beta_{2 k+1, n}$ with $j=n$; $d_{k, n}=\gamma_{2 k, n}$ with $j=n ; d_{k, n}=\delta_{k, n}$ with $j=2 n+2 ; d_{k}=\alpha_{k} ; d_{k}=\beta_{2 k+1}$; $d_{k}=\gamma_{2 k} ; d_{k}=\delta_{k}$.

Proof. If $c_{n}$ denotes the coefficient of $z$ in the Taylor expansion of $\log R_{n}(z)$ about the origin, then $\left|c_{n}\right| \rightarrow K<\infty$ as $n \rightarrow \infty$ and thus $-\infty<c_{n}<\sum_{k=1}^{n}\left(-1 / \alpha_{k, n}\right)-1 / \beta_{1, n}-2 \sum_{k=1}^{n}\left(1 / \beta_{2 k+1, n}\right)<0$. This establishes the first two cases. The third and fourth follow from the 
relations $0<\beta_{2 k-1}<\gamma_{2 k, n}$ and $0<\alpha_{k, n}<\delta_{k+1, n}$. The remaining cases follow from Lemma 5 .

LEMMA 7. If

$$
\pi(z)=\left[1 /\left(1+z / \gamma_{1}\right)^{2}\right]\left[\prod_{k=1}^{\infty} \alpha_{k}^{*} \prod_{k=0}^{\infty} \beta_{2 k+1}^{*} \prod_{k=1}^{\infty}\left(\gamma_{2 k}^{*}\right)^{3}\right],
$$

where $\alpha_{k}^{*}=1-z / \alpha_{k}, \beta_{2 k+1}^{*}=1-z / \beta_{2 k+1}$, and $\gamma_{2 k}^{*}=1-z / \gamma_{2 k}$, then $f^{\prime}(z)$ $=\exp \left(\delta^{\prime} z\right) \pi(z)$ where $s_{n}^{\prime} \rightarrow \delta^{\prime}$ as $n \rightarrow \infty$ with $s_{n}^{\prime}$ the coefficient of $z$ in the Taylor expansion of $\log \left[R_{n}^{\prime}(z) / \pi(z)\right]$ about the origin.

Proof. Because of the ordering of the $\alpha$ 's, $\beta$ 's, and $\gamma^{\prime}$ 's and because of Lemmas 5 and $6, \log \left[R_{n}^{\prime}(z) \pi(z)\right] \rightarrow \delta^{\prime} z$ uniformly on any compact subset of the plane as $n \rightarrow \infty$.

\section{LEMMA $8 . \delta^{\prime} \leqq 0$.}

Proof. Because the factors of $\pi(z)$ are canonical products of genus zero, for every $\epsilon>0$ and $0<\rho \leqq|\arg z| \leqq \pi-\rho, \pi(z)=O[\exp (\epsilon|z|)]$ and $1 / \pi(z)=O[\exp (\epsilon|z|)]$. Then for $\arg z$ satisfying these conditions and $|z|>R$ sufficiently large, $\exp \left[\delta^{\prime} R(z)-\epsilon|z|\right] \leqq\left|f^{\prime}(z)\right| \leqq$ $\exp \left[\delta^{\prime} R(z)+\epsilon|z|\right]$. If $\delta^{\prime}>0$ and $|z|>R$, there exists $\phi_{1}>0$ such that $\left|f^{\prime}(z)\right| \geqq \exp \left[\phi_{1}|z|\right]$ for $\pi / 6 \leqq \arg z \leqq \pi / 3$ and $\left|f^{\prime}(z)\right| \leqq \exp \left[-\phi_{1}|z|\right]$ for $2 \pi / 3 \leqq \arg z \leqq 5 \pi / 6$. If $\delta^{\prime}<0$ and $|z|>R$, there exists $\phi_{2}>0$ such that $\left|f^{\prime}(z)\right| \leqq \exp \left[-\phi_{2}|z|\right]$ for $\pi / 6 \leqq \arg z \leqq \pi / 3$ and $\left|f^{\prime}(z)\right|$ $\geqq \exp \left[\phi_{2}|z|\right]$ for $2 \pi / 3 \leqq \arg z \leqq 5 \pi / 6$.

Since the distance from the origin to $C_{n+1} \rightarrow \infty$ as $n \rightarrow \infty$, then there exists a sequence of positive numbers $\left\{r_{n}\right\}$ such that $r_{n} \rightarrow \infty$ as $n \rightarrow \infty$ and such that for $z$ on $C_{n+1},|z|>r_{n}$. Let $z_{1,2 j+1}$ and $z_{2,2 j+1}$ be points of $C_{2 j+1}$ such that $\arg z_{1,2 j+1}=\pi / 6$ and $\arg z_{2,2 j+1}=\pi / 3$. Let $z_{1,2 j}$ and $z_{2,2 j}$ be points of $C_{2 j}$ such that $\arg z_{1,2 j}=5 \pi / 6$ and arg $z_{2,2 j}=2 \pi / 3$.

If $\delta^{\prime}>0$, then for $\zeta_{1}, \zeta_{2} \in\{\zeta|2 \pi / 3 \leqq \arg \zeta \leqq 5 \pi / 6,| \zeta \mid>R\}$, $\left|f\left(\zeta_{2}\right)-f\left(\zeta_{1}\right)\right| \leqq \exp \left[-\phi_{1} R\right]\left|\zeta_{2}-\zeta_{1}\right|$, and thus $f(z) \rightarrow k$, a constant, uniformly in $\{z \mid 2 \pi / 3 \leqq \arg z \leqq 5 \pi / 6\}$ as $z \rightarrow \infty . k=0$, because as $z \rightarrow \infty$, say along the ray arg $z=3 \pi / 4, f(z)<0$ where the ray crosses $C_{2 n}$ and $f(z)>0$ where the same ray crosses $C_{2 n+1}$. Thus for $j$ sufficiently large, $0<f\left(z_{2,2 j+1}\right)<1$. Now for $|z| \geqq r_{2 j+1}$ sufficiently large, $b_{2 j+1}-a_{2 j+1} \geqq f\left(z_{2,2 j+1}\right)-f\left(z_{1,2 j+1}\right) \geqq \exp \left[\phi_{1} r_{2 j+1}\right] r_{2 j+1} \pi / 6$, and hence each of these expressions tends to $\infty$ as $j \rightarrow \infty$. Because $f\left(z_{1,2 j+1}\right)>0$, then $f\left(z_{2,2 j+1}\right) \rightarrow \infty$ as $j \rightarrow \infty$, which is a contradiction and shows $\delta^{\prime} \ngtr 0$.

However, if $\delta^{\prime}<0$, then as $z \rightarrow \infty$, say along arg $z=\pi / 4$, then $|f(z)| \rightarrow 0$, while as $j \rightarrow \infty,\left|f\left(z_{2,2 j}\right)-f\left(z_{1,2 j}\right)\right| \rightarrow \infty$ where $f\left(z_{2,2 j}\right)<0$ and thus $f\left(z_{1,2 j}\right) \rightarrow-\infty$ as $j \rightarrow \infty$. 
Lemma 9. If $\delta^{\prime}<0$, then $a_{n}=o(1)$ as $n \rightarrow \infty$ and there exist positive constants $p$ and $A$ such that for $n$ sufficiently large, $b_{2 n+1} \geqq A \exp [p n]$.

Proof. For $\zeta_{n}$ a point of $C_{n}$ and $\pi / 6 \leqq \arg \zeta_{n} \leqq \pi / 3,0<a_{n}<\left|f\left(\zeta_{n}\right)\right|$, and from the proof of Lemma $8,\left|f\left(\zeta_{n}\right)\right| \rightarrow 0$ as $n \rightarrow \infty$. If $z_{1,2 j+1}$ and $z_{2,2 j+1}$ are points of $C_{2 j+1}$ such that arg $z_{1,2 j+1}=5 \pi / 6$ and arg $z_{2,2 j+1}$ $=2 \pi / 3$, then $b_{2 j+1}-a_{2 j+1} \geqq \exp \left[\phi_{2} r_{2 j+1}\right] r_{2 j+1} \pi / 6 \geqq A \exp [p n]$.

LEMma 10. If $P(z)=\left[z /\left(1+z / \gamma_{1}\right)\right]\left[\prod_{k=2}^{\infty} \delta_{k}^{*} \prod_{k=1}^{\infty}\left(\gamma_{2 k}^{*}\right)^{2}\right]$, where $\delta_{k}^{*}=1-z / \delta_{k}$, then $f(z)=\exp [\delta z] P(z)$ where $s_{n} \rightarrow \delta$ as $n \rightarrow \infty$ with $s_{n}$ the coefficient of $z$ in the Taylor expansion of $\log \left[R_{n}(z) / P(z)\right]$ about the origin.

ProOF. $\log \left[R_{n}(z) / P(z)\right] \rightarrow \delta z$ uniformly on any compact subset of the plane as $n \rightarrow \infty$.

Lemma 11. $\delta=\delta^{\prime}$.

Proof. Because of Lemma 5, as $n \rightarrow \infty$, $0 \leqq \lim \sup \left|s_{n}-s_{n}^{\prime}\right|$

$$
\begin{aligned}
& \leqq \\
& \leqq \\
& \quad \lim \sup \left|\sum_{k=m}^{n}\left(1 / \gamma_{2 k, n}-1 / \beta_{2 k+1, n}\right)\right|+\lim \sup \left|\sum_{k=m}^{\infty}\left(1 / \gamma_{2 k}-1 / \beta_{2 k+1}\right)\right| \\
& \quad+\lim \sup \left|\sum_{k=m}^{2 n+2}\left(1 / \delta_{k, n}\right)-\sum_{k=m}^{2 n+1} 1 / \alpha_{k, n}\right|+\lim \sup \left|\sum_{k=m}^{\infty}\left(1 / \delta_{k}-1 / \alpha_{k}\right)\right| \\
& \leqq \lim \sup 1 / \gamma_{m, n}+1 / \gamma_{m}+\lim \sup 1 / \delta_{m, n}+1 / \delta_{m}=2\left(\gamma_{m}+\delta_{m}\right),
\end{aligned}
$$

for every $m \geqq 2$.

Lemma 12. If $\delta<0$, then there exists a positive constant $p$ such that $a_{n}=O[\exp (-p n)]$ as $n \rightarrow \infty$.

Proof. $|f(z)|$ satisfies the same inequalities as $\left|f^{\prime}(z)\right|$ in the proof of Lemma 8. Thus if $\delta<0$, then there exists a positive constant $p$ such that for $z_{n}$ on $C_{n}$ and

$$
\arg z_{n}=\pi / 4,0<a_{n}<\left|f\left(z_{n}\right)\right|=O[\exp (-p n)] .
$$

Theorem. $A$ surface of the class defined is parabolic and the mapping function is given by $f(z)=\exp [\delta z] P(z)$ where $f^{\prime}(z)=\exp [\delta z] \pi(z)$. If $\delta<0$, then there exist positive constants $A$ and $p$ such that $a_{n}$ $=O[\exp (-p n)]$ as $n \rightarrow \infty$ and $b_{2 n+1} \geqq A \exp [p n]$ for $n$ sufficiently large. Also, $\sum_{k=2}^{\infty} 1 / d_{k}<\infty$ for $d_{k}=\alpha_{k}, d_{k}=\beta_{2 k+1}, d_{k}=\gamma_{2 k}$, and $d_{k}=\delta_{k}$.

The remaining lemmas are proof of the following theorem.

THEOREM. Let $f(z)=\exp [\delta z]\left[z /\left(1+z / \gamma_{1}\right)\right] \coprod_{k=1}^{*}\left[\delta_{2 k}^{*} \delta_{2 k+1}^{*} /\left(\gamma_{2 k}^{*}\right)^{2}\right]$ 
where $\delta_{2 k}^{*}=1-z / \delta_{2 k}, \delta_{2 k+1}^{*}=1-z / \delta_{2 k+1}, \gamma_{2 k}^{*}=1-z / \gamma_{2 k}, \sum_{k=1}^{\infty} 1 / \delta_{k}<\infty$, $\sum_{k=1}^{\infty} 1 / \gamma_{2 k}<\infty, \delta \leqq 0$, and for $n \geqq 1,0<\delta_{2 n}<\delta_{2 n+1}<\delta_{2 n+2}, 0<\gamma_{1}<\gamma_{2 n}$ $<\gamma_{2 n+2}$. The Riemann surface of the inverse of $f(z)$ is a surface of the class defined.

Lemma 13. Suppose $\delta=0$. For $n>2$ and $n$ an odd integer, $R_{n}(z)$ $=\left[z /\left(1+z / \gamma_{1}\right)\right] \prod_{k=1}^{(n-1) / 2}\left[\left(\delta_{2 k}^{*}\right)\left(\delta_{2 k+1}^{*}\right) /\left(\gamma_{2 k}^{*}\right)^{2}\right]$ has the following properties: (a) $R_{n}(z) \rightarrow f(z)$ uniformly on any compact subset of the plane as $n \rightarrow \infty$. (b) the curves other than the real axis on which $R_{n}(z)$ is real are $n-1$ simple, closed, nonintersecting curves about the origin.

Proof. The truth of (a) is obvious. By applying Rolle's Theorem to $R_{n}(x)$ for $x$ real, a zero $\alpha_{k, n}$ of $R_{n}^{\prime}(z)$ is determined between each pair of zeros of $R_{n}(z)$, and a zero $\beta_{k, n}$ of $R_{n}^{\prime}(z)$ is determined between each pair of $\gamma$ 's. These critical points together with the $\gamma$ 's account for the exact total branch order of $2 n-2$ for $R_{n}(z)$. Then through each of the $\alpha$ 's, $\beta$ 's, and $\gamma$ 's passes a curve, in addition to the real axis, on which $R_{n}(z)$ is real. Because $R_{n}(\bar{z})=\bar{R}_{n}(z)$, these curves are symmetric about the real axis, and because $\infty$ is not a critical point the curves are simple, closed, nonintersecting ones, each of which intersects the real axis at two points. Furthermore, because of the nonintersecting property and because of the behavior of $R_{n}(z)$, no curve can intersect two $\alpha$ 's, and thus a curve $C_{1, n}$ intersects the real axis in $\alpha_{1, n}$ and $-\beta_{1, n}$, curves $C_{2 k, n}$ intersect the real axis in $\alpha_{2 k, n}$ and $-\gamma_{2 k}$, and curves $C_{2 k+1, n}$ intersect the real axis in $\alpha_{2 k+1, n}$ and $-\beta_{2 k+1, n}$.

Lemma 14. The points of $C_{k, n}$ tend to the points of a curve $C_{k}$ as $n \rightarrow \infty$, where $C_{k}$ intersects the real axis twice and does not pass through $\infty$.

Proof. By Hurwitz's theorem, $\alpha_{k, n} \rightarrow \alpha_{k}$ and $\beta_{k, n} \rightarrow \beta_{k}$ as $n \rightarrow \infty$. Hence $C_{k}$ intersects the real axis at $\alpha_{k}$ and $-\beta_{k}$ or $-\gamma_{k}$. Suppose there exists a curve $C_{k}$ which has $z=\infty$ as one of its points, and let $C_{m}$ be the curve with minimum subscript such that $C_{m}$ has $z=\infty$ as one of its points. As $z \rightarrow \infty$ from $\alpha_{m}$ along the upper half of $C_{m}$, $f(z) \rightarrow \infty$ because otherwise the upper parts of $C_{m}$ and $C_{m+1}$ and the segment $\left(\alpha_{m}, \alpha_{m+1}\right)$ bound a region on whose boundary $f(z)$ steadily increases or decreases from $f\left(\alpha_{m}\right)$ back to this value, which is impossible. As $z \rightarrow \infty$ from its intersection with the negative real axis along the upper part of $C_{m}, f(z) \rightarrow \infty$, because if $f(z) \rightarrow k<\infty$, a contradiction arises (see p. 67, [3]). If $C_{k}$ is the curve with minimum even subscript such that $C_{k-1}$ has $z=\infty$ as one of its points, then the behavior of $f(z)$ on the boundary of the region in the upper half of the plane and bounded by $C_{k-1}, C_{k}$, and the segment $\left(-\gamma_{k},-\beta_{k-1}\right)$ is impossible. 
LEMMA 15. $f(z)$ is a schlicht and conformal map of the upper half of the annular region between $C_{j}$ and $C_{j+1}$ onto $g(-1)^{i}(w)>0$.

Proof. This is an immediate consequence of Darboux's theorem.

Lemma 16. If $\delta<0$ and $\lambda_{n}$ is an integer such that $\lambda_{n} /-\delta>\delta_{n}$, then

$$
R_{n}(z)=\left[\left(1+\delta z / \lambda_{n}\right)^{\lambda_{n}}\right]\left[z /\left(1+z / \gamma_{1}\right)\right]\left[\prod_{k=1}^{(n-1) / 2}\left(\delta_{2 k}^{*}\right)\left(\delta_{2 k+1}^{*}\right) /\left(\gamma_{2 k}^{*}\right)^{2}\right] \text {, }
$$

for $n>2$ and $n$ an odd integer, is a rational function with properties similar to those of Lemmas 13 through 15.

Proof. The proofs are immediate, except for the property of Lemma 14 possibly. In this case the proof on page 67 of [4] can be used.

\section{REFERENCES}

1. C. Carathéodory, Untersuchungen über die konformen Abbildungen von festen und varänderlichen Gebieten, Math. Ann. vol. 72 (1912) pp. 107-144.

2. G. R. MacLane, Riemann surfaces and asymptotic values associated with real entire functions, The Rice Institute Pamphlet, 1952.

3. R. Nevanlinna, Eindeutige Analytische Funktionen, Berlin, Springer, 1953.

4. H. E. Taylor, Determination of the type and properties of the mapping function of a class of doubly connected Riemann surfaces, Proc. Amer. Math. Soc. vol. 4 (1953) pp. $52-68$.

The Rice Institute AND

The UNIVERsity of TeXas 